\title{
Optimal placement of piezoelectric actuators and polyvinylidene fluoride error sensors in active structural acoustic control approaches
}

\author{
Robert L. Clark and Chris R. Fuller \\ Mechanical Engineering Department, Virginia Polytechnic Institute and State University, Blacksburg, \\ Virginia 24061
}

(Received 14 August 1991; revised 2 March 1992; accepted 16 April 1992)

Optimization of the location of a rectangular piezoelectric actuator and both the size and location of a rectangular surface strain error sensor constructed from polyvinylidene fluoride (PVDF) for active structural acoustic control (ASAC) is studied in this work. An algorithm is proposed for choosing the optimal actuator/sensor configuration for controlling sound from a baffled simply supported plate excited harmonically, and the resulting acoustic response is predicted from analytical models. These results are compared to those measured in the lab on a test rig duplicating the appropriate boundary conditions and situated in an anechoic chamber. Results from a single optimally located control actuator are compared to those from control with a nonoptimally positioned actuator as well as multiple control actuators. In addition, either microphones are used to provide error information in the test cases or a single optimally located and dimensioned PVDF error sensor is implemented as the cost function. Results from this study indicate that optimization of control actuators and error sensors provides a method for realizing adaptive structures for active structural acoustic control (ASAC), rivaling in importance the performance increases gained when acoustic control is achieved with microphone error sensors and multiple control actuators.

PACS numbers: $43.40 . \mathrm{Vn}, 43.50 . \mathrm{Ki}, 43.40 . \mathrm{Yq}$

\section{INTRODUCTION}

Active structural acoustic control (ASAC) has taken many directions in recent years due to advances in digital signal processing boards as well as actuator and sensor materials. The objective today is not simply to control the sound radiation from the structure, but to develop adaptive structures. An adaptive structure is one with both the actuators and sensors either embedded in the structure or bonded to the surface such that the structural response can be modified with varying input disturbances. Piezoelectric materials such as PZT and polyvinylidene fluoride (PVDF) have been used extensively as actuators and sensors for controlling vibration of structures. ${ }^{1-8}$ In addition, many studies have been devoted to the control of sound radiation from vibrating structures with piezoelectric actuators bonded to the surface of the structure. ${ }^{9-13}$ Dynamic models of the response characteristics of piezoelectric actuators provided the foundation for their implementation in active structural acoustic control as well as active vibration control, ${ }^{1-3,14}$ and experiments tailored to evaluate these models confirmed that they were useful for this purpose. ${ }^{1,15,16}$ While much research has been dedicated to designing shaped sensors with polyvinylidene fluoride (PVDF) for vibration control, ${ }^{3,4,6-8}$ only recently has the material been suggested as a sensor for structureborne sound. ${ }^{12,13}$

Since the output of the PVDF sensor is proportional to the integral of strain over the area of application, the material is well suited for vibration control; however, in controlling structure-borne sound the cost function is typically con- structed from variables related to radiation of sound, which complicates the choice of sensor shape and location. A sensor could be designed such that the total structural response (i.e., integral of the surface strain over the area of sensor application) is the cost function, but this would require a significant number of control channels and authority, specifically for off-resonance response when multiple modes of the structure contribute. In controlling the vibration of a structure, every mode contributing to the structural response must be minimized; however, in controlling structure-borne sound, only those modes with high radiation efficiency (or correspondingly the supersonic wave-number components) must be controlled to obtain significant levels of far-field acoustic attenuation. This important concept can lead to a reduction in the level of control complexity and thus reduce the number of control channels required to obtain the desired levels of far-field acoustic attenuation.

Recent analytical studies by Wang and Fuller have been devoted to optimizing the location of piezoelectric actuators on the surface of the structure for minimizing far-field structure-borne sound. ${ }^{17}$ Since an acoustic objective function was chosen, the optimal location of the actuator is such that control authority over the more efficient acoustic radiators is obtained. If this concept is applied to structural sensors such as PVDF, the optimal location of a rectangular element of PVDF bonded to the surface of a structure can be determined such that the acoustic objective function is minimized. Upon accomplishing this task, the elements necessary to create an adaptive structure for controlling structureborne sound are in place. As opposed to requiring micro- 
phones located in the acoustic far field as error sensors, the structure is equipped with a sensor bonded to the surface that yields equivalent error information.

The purpose of this work is to both analytically and experimentally demonstrate the advantages afforded with optimization of both piezoelectric actuators and polyvinylidene fluoride error sensors in active structural acoustic control. For the purpose of this study, a baffled, simply supported, rectangular plate is implemented as the test structure example since an analytical solution for both the acoustic response and structural response can be readily computed. In both the analytical study and the experiments, the plate is excited by a harmonic point force disturbance, and control is achieved with piezoelectric actuators and PVDF error sensors. Linear quadratic optimal control theory was used to determine the analytical response, while the filtered-x version of the adaptive LMS algorithm was implemented on a TMS320C25 digital signal processing board to achieve control experimentally. ${ }^{11}$

The theory required to determine the optimal actuator and error sensor location are outlined, and several test cases are chosen to demonstrate the level of control achievable. Both experimental and analytical results are presented for optimally located actuator/error sensor configurations and multi-channel nonoptimal configurations. By comparing the experimental and analytical results, the design approach implemented in the optimization routine was evaluated. In addition, to demonstrate the advantages afforded with optimization, results comparing optimally located actuator/sensor configurations are compared to those for multi-channel nonoptimal configurations. Results from this study demonstrate that a single, optimally located, piezoelectric actuator and PVDF error sensor result in reduction of structureborne sound rivaling that achieved with three arbitrarily located actuators and three microphones located in the acoustic field as error sensors.

\section{THEORY}

The optimal location of single or multiple piezoelectric actuators for controlling structure-borne sound was theoretically obtained as previously outlined by Wang and Fuller utilizing an acoustic objective function consisting of a finite number of microphones approximating the total radiated acoustic power. ${ }^{18}$ This approximation of the objective function is much less computationally intensive and yields optimal designs approaching that achieved when using the radiated acoustic power as the objective function. A flow chart of the solution strategy, which is similar to that presented earlier by Wang and Fuller is presented in Fig. $1 .{ }^{17}$ As illustrated, two alternative paths exist in the flow chart. In the first path, the optimal location of the control actuator(s) is determined, and upon converging to a solution meeting the required accuracy test, the second path of the algorithm is executed. In this path, the optimal size and location of a rectangular PVDF error sensor(s) is determined with the same acoustic objective function and the previously computed coordinates for the optimal location of the rectangular control actuator(s).

The core algorithm for determining the optimal piezoelectric actuator location(s) or the optimal PVDF error sensor size(s) and location(s) is identical. Linear quadratic optimal control theory is used to compute the optimal control solution for the given control actuator/error sensor loca-

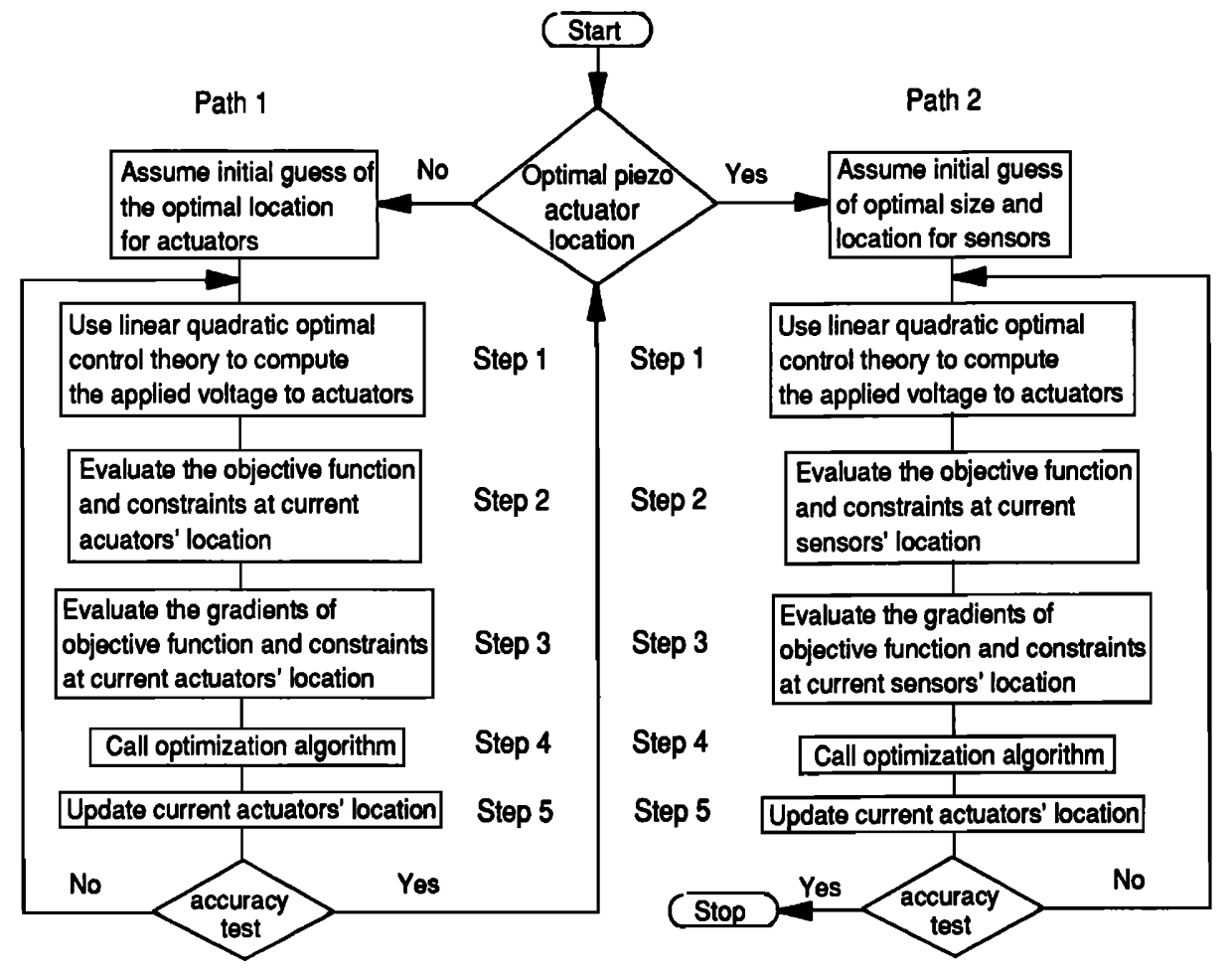

FIG. 1. Flow chart of optimization algorithm. 
tion(s) in step 1, regardless of whether path 1 or path 2 is taken. Upon determining the optimal solution for the given actuator/sensor configuration(s), the objective function and constraints are evaluated in step 2 . In step 3, the gradients of the objective function and constraints are computed for the current actuator location(s) or current error sensor size(s) and location(s), depending on whether path 1 or path 2 is taken, respectively. Upon determining the gradients, the optimization algorithm is invoked in step 4, and the actuator or sensor design parameters are updated depending on the chosen path. The accuracy test is completed based upon the updated parameters and the program either terminates or proceeds.

The distinguishing feature of the algorithm presented is that the optimal control voltages are obtained by linear quadratic optimal control theory (LQOCT). This method of solution was chosen to reduce numerical difficulty and consequently computational time. To clarify the optimization approach, step 1 of the algorithm is outlined in Sec. I A to describe the method of implementing linear quadratic optimal control both for path 1 and path 2 of Fig. 1 . The remaining steps of the algorithm are outlined in Sec. I B to provide the necessary information for determining the optimal actuator/sensor configuration. Since the core algorithm is identical for optimization of actuator location (s) or sensor size(s) and location(s), the following description applies to either path 1 or path 2 of the flow chart.

\section{A. Linear quadratic optimal control theory}

In developing the analytical model for the purpose of this study, one must derive the response of the plate to a point force representing the disturbance, and a piezoelectric actuator representing the control input. In addition, analytical models with a computed response proportional to the response of the chosen error sensors (i.e., microphones and PVDF strip sensors) must be derived. After developing the sensor and actuator equations, the control approach must be modeled mathematically. For this particular study, linear quadratic optimal control was chosen to obtain the optimal control voltage at the piezoelectric actuator. To convey this information, subsections are devoted to actuator models, sensor models and control approaches. Due to constraints on the length of this paper, only the necessary equations to construct the computational model are presented; however, references are included where the reader can find expanded discussions on the components.

\section{Actuator equations}

As stated earlier, the structure chosen for this particular study is a simply supported plate with the following response from a modal decomposition: ${ }^{19}$

$$
w(x, y, t)=\sum_{m=1}^{\infty} \sum_{n=1}^{\infty} W_{m m} \sin \left(\gamma_{m} x\right) \sin \left(\gamma_{n} y\right) \exp (j \omega t),
$$

where $\gamma_{m}=m \pi / L_{x}, \gamma_{n}=n \pi / L y, W_{m n}=$ modal amplitudes, $L_{x}=$ length of plate in $x$ direction, $L_{y}=$ length of plate in $y$ direction, $\omega=$ driving frequency, $t=$ time.

Solving the equation of motion for the plate subjected to harmonic excitation by a point force located at $x-y$ coordinates $(c, d)$ yields the following expression for the modal amplitudes (including proportional damping):

$$
F_{m n}=\frac{4 F}{\rho L_{x} L_{y}} \frac{\sin \left(\gamma_{m} c\right) \sin \left(\gamma_{n} d\right)}{\left(\omega_{m n}^{2}-\omega^{2}+j 2 \eta \omega \omega_{m n}\right)} .
$$

The resonant frequencies of the simply supported plate expressed in the previous equation are given by

$$
\omega_{m n}^{2}=\left(D_{e} / \rho\right)\left[\left(\gamma_{m}\right)^{2}+\left(\gamma_{n}\right)^{2}\right]^{2},
$$

where the plate flexural stiffness is defined by

$$
D_{e}=\frac{E h^{3}}{12\left(1-v^{2}\right)},
$$

and $E=$ Young's modulus of plate, $h=$ thickness of plate, $v=$ Poisson's ratio of plate, $\eta=$ damping ratio, $\rho=$ mass density of plate per unit area.

If we solve the equation of motion for the plate with two piezoelectric patches bonded symmetrically to the front and back surface of the plate and electrically wired out of phase to induce uniform bending about the central axis of the plate, the resulting modal amplitudes can be derived (including proportional damping): ${ }^{14}$

$$
\begin{aligned}
P_{m n}^{p}= & \frac{4 C_{0} \epsilon_{p e}}{\rho L_{x} L_{y}\left(\omega_{m n}^{2}-\omega^{2}+j 2 \eta \omega_{m n} \omega\right)} \\
& \times\left(-\frac{\gamma_{m}^{2}+\gamma_{n}^{2}}{\gamma_{m} \gamma_{n}}\left[\cos \left(\gamma_{m} x_{1}^{p}\right)-\cos \left(\gamma_{m} x_{2}^{p}\right)\right]\right. \\
& \left.\times\left[\cos \left(\gamma_{n} y_{1}^{p}\right)-\cos \left(\gamma_{n} y_{2}^{p}\right)\right]\right) .
\end{aligned}
$$

Constants used in the above equation are defined as follows:

$$
\begin{aligned}
& \epsilon_{p e}=d_{31} V^{p} / t, \\
& C_{0}=-\frac{E h}{6} \frac{(1+v)}{(1-v)} \frac{P}{\left[1+v-\left(1+v_{p e}\right) P\right]}, \\
& P=-\frac{E_{p e}}{E} \frac{\left(1-v^{2}\right)}{\left(1-v_{p e}^{2}\right)} K, \\
& K=\frac{6 t h(h+t)}{\left(h^{3}+8 t^{3}\right)+6 h t^{2}},
\end{aligned}
$$

where $\left(x_{1}^{p}, y_{1}^{p}\right)=$ coordinates of lower left corner of $p^{\text {th }}$ actuator, $\left(x_{2}^{p}, y_{2}^{p}\right)=$ coordinates of upper right corner of $p^{\text {th }}$ actuator, $d_{31}=$ piezoelectric strain constant, $V^{p}=$ complex voltage of $p^{\text {th }}$ actuator, $t=$ thickness of piezopatch, $E_{p e}$ $=$ Young's modulus of piezopatch, $v_{p e}=$ Poisson's ratio of piezopatch.

To obtain the combined response due to excitation by the point force disturbance and multiple piezoelectric actuators, superposition is applied. Based on Eq. (1), the modal amplitude for the summed response can be written as below:

$$
W_{m n}=F_{m n}+\sum_{p=1}^{n_{a}} P_{m n}^{p},
$$

where $n_{a}=$ number of actuators.

\section{Sensor models}

In experiments previously performed, ${ }^{12,13}$ two types of sensors were implemented in the control approach, PVDF 
strip sensors and microphones. Lee and Moon presented an equation representing the electrical response of PVDF as a result of strain induced in the structure. ${ }^{8}$ This expression was formulated in terms of charge, and the resulting voltage can be obtained by dividing the expression by the sensor capacitance. The electrical response of each PVDF error sensor must be conditioned with a voltage amplifier having an input impedance several orders of magnitude greater than that of the sensor. This isolates the electrical response of the sensor from the relatively low impedance $(10 \mathrm{k} \Omega)$ analog to digital input board. With this said, the expression for the response (in terms of voltage) of a rectangular strip of PVDF film distributed over the surface of the simply supported plate can be written as follows:

$$
\begin{aligned}
V_{u}(t)= & \frac{\left(h+t_{p}\right) t_{p}}{2 \epsilon A} \sum_{m=1}^{\infty} \sum_{n=1}^{\infty} W_{m n}\left(e_{31}^{0} \frac{m L_{y}}{n L_{x}}+e_{32}^{0} \frac{n L_{x}}{m L_{y}}\right) \\
& \times\left[\cos \left(\gamma_{m} x_{4}^{u}\right)-\cos \left(\gamma_{m} x_{3}^{u}\right)\right] \\
& \times\left[\cos \left(\gamma_{n} y_{4}^{u}\right)-\cos \left(\gamma_{n} y_{3}^{u}\right)\right] \exp (j \omega t),
\end{aligned}
$$

where $\left(x_{3}^{u}, y_{3}^{u}=\right.$ coordinates of lower left corner of $u$ th PVDF strip, $\left(x_{4}^{u}, y_{4}^{u}=\right.$ coordinates of upper right corner of $u$ th PVDF strip, $e_{31}^{0}=$ sress per charge $\left(65.3 E-3 \mathrm{C} / \mathrm{m}^{2}\right)$ in $x$ direction, $e_{32}^{0}=$ stress per charge $\left(38.7 E-3 \mathrm{C} / \mathrm{m}^{2}\right)$ in $y$ direction, $\epsilon=$ permitivity of PVDF, $A=$ area of sensor, $h=$ thickness of plate, $t_{p}=$ thickness of PVDF.

Observe that while the previous equation represents the response of a single rectangular strip of PVDF, sensors of arbitrary shape can be modeled with this equation by simply dividing the irregular shape into subdivisions of rectangular elements and summing the response from each element, with the correct phasing, similar to numerical integration.

If a microphone is chosen as the error sensor, an expression for the sound pressure radiated from the simply supported plate can be used in developing the cost function to model the sensor. ${ }^{20}$ Rayleigh's integral is evaluated at a radius of $1.6 \mathrm{~m}$, which corresponds to the radius of the traversing array in the anechoic chamber. This radius does not satisfy the far-field conditions and thus requires a numerical solution of the Rayleigh integral for the purpose of comparing experimental and analytical results. To this end, the pressure can be written as a function of the modal response of the plate as follows (where the harmonic time dependence has been omitted for convenience):

$$
\begin{aligned}
p\left(x^{\prime}, y^{\prime}, z^{\prime}\right)= & -\frac{\omega^{2} \rho_{0}}{2 \pi} \sum_{n=1}^{N} \sum_{m=1}^{M} W_{m n} \\
& \times \int_{0}^{L_{x}} \int_{0}^{L_{y}} \sin \left(\frac{m \pi x}{L_{x}}\right) \sin \left(\frac{n \pi y}{L_{y}}\right) \\
& \times \frac{\exp \left[-j k \sqrt{\left(x^{\prime}-x\right)^{2}+\left(y^{\prime}-y\right)^{2}+\left(z^{\prime}\right)^{2}}\right]}{\sqrt{\left(x^{\prime}-x\right)^{2}+\left(y^{\prime}-y\right)^{2}+\left(z^{\prime}\right)^{2}}} \\
& \times d y d x,
\end{aligned}
$$

where $\left(x^{\prime}, y^{\prime}, z^{\prime}\right)=$ spatial coordinates of pressure, $k=$ wave number in fluid medium (air), $c=$ speed of sound in fluid medium (air), $\rho_{0}=$ density of fluid medium (air).

By setting $y=y-L_{y} / 2$ and $x=x-L_{x} / 2$ and changing the limits of integration correspondingly in Eq. (12), the

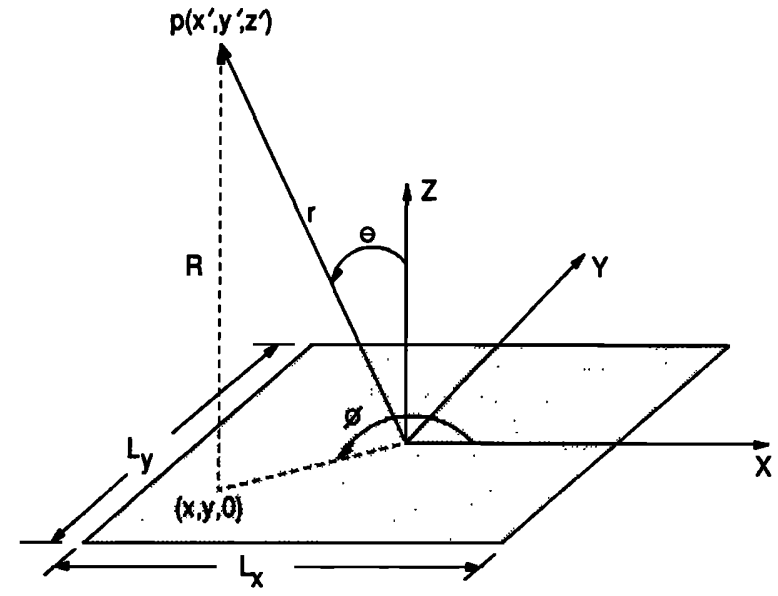

FIG. 2. Coordinate system for plate acoustic response.

coordinates for computing the pressure are transformed to the center of the plate. A schematic of the coordinate system is presented in Fig. 2 with respect to both rectangular and spherical coordinates.

\section{Control approach}

Linear quadratic optimal control was utilized to compute the optimal control voltages for each piezoelectric actuator at the chosen location. In this approach, the cost function (function to be minimized) is formulated as the square of the chosen error sensors.

$$
C=\sum_{b=1}^{n_{e}} q_{b} q_{b}^{*},
$$

where $q_{b}^{*}$ is the complex conjugate of $q_{b}$,

$$
q_{b}=\left(\sum_{s=1}^{n_{a}} T_{b s} \hat{V}_{s}\right)+T_{b D} F,
$$

where $T_{b s}=$ transfer function between the sth actuator and the $b$ th error sensor, $\hat{V}_{s}=$ complex voltage of sth actuator, $T_{b D}=$ transfer function between the disturbance and the $b$ th actuator, $F=$ input force, $n_{a}=$ number of control actuators.

Taking the partial derivative of the cost function with respect to the real and imaginary part of each control voltage results in the following system of linear equations that must be solved:

$$
\left[\sum_{b=1}^{n_{e}}\left(\sum_{l=1}^{n_{a}} T_{b l} \widehat{V}_{l}+T_{b D} F\right) T_{b s}^{*}=0\right], \quad s=1, n_{a} .
$$

The previous system of equations can be represented with linear algebra as follows:

$$
[A][\hat{V}]=[b] \text {. }
$$

For example, if $n_{e}=3$ and $n_{a}=2$, then

$$
\begin{aligned}
& {[\hat{V}]=\left[\begin{array}{l}
V_{1} \\
V_{2}
\end{array}\right],} \\
& {[b]=\left[\begin{array}{l}
-\left(T_{1 D} T_{11}^{*}+T_{2 D} T_{21}^{*}+T_{3 D} T_{31}^{*}\right) F \\
-\left(T_{1 D} T_{12}^{*}+T_{2 D} T_{22}^{*}+T_{3 D} T_{32}^{*}\right) F
\end{array}\right],}
\end{aligned}
$$


and

$$
[A]=\left[\begin{array}{cc}
\left(T_{11} T_{11}^{*}+T_{21} T_{21}^{*}+T_{31} T_{31}^{*}\right) & \left(T_{12} T_{11}^{*}+T_{22} T_{21}^{*}+T_{32} T_{31}^{*}\right) \\
\left(T_{11} T_{12}^{*}+T_{21} T_{22}^{*}+T_{31} T_{32}^{*}\right) & \left(T_{12} T_{12}^{*} T_{22} T_{22}^{*}+T_{32} T_{32}^{*}\right)
\end{array}\right] .
$$

Since the number of unknowns is equivalent to the number of equations, the solution can be obtained as follows as long as the matrix $[A]$ is nonsingular: ${ }^{12}$

$$
[\hat{V}]=[A]^{-1}[b] .
$$

While the above equation yields the optimal solution, it may not be the most computationally stable method of solving for the complex control voltages. In addition, the number of error sensors must be greater than or equal to the number of actuators or the system of equations is under determined.

If path 1 of the algorithm depicted in Fig. 1 is executed, the error sensors chosen in the linear quadratic optimal control solution are microphones with coordinates corresponding to those implemented in the nonlinear optimization algorithm of step 4. Microphones were chosen in this path since they provide the best estimate of the desired cost function (i.e., far-field sound radiation) and as previously discussed, the number of error sensors must be greater than or equal to the number of control actuators to yield a unique solution to Eq. (16). Upon converging to a solution, the optimal location(s) of the control actuator(s) for minimizing far-field sound radiation is obtained. If path 2 of the algorithm is executed, the piezoelectric actuator location(s) obtained from path 1 is used as the control input, and as opposed to microphone error sensors, the PVDF error sensor(s) is implemented in the linear quadratic optimal control since the location (s) and dimensions of the rectangular sensor(s) are the variables to be optimized. Note that in both cases, the objective function for the nonlinear optimization is an approximation of the far-field sound power radiated based upon evaluating the pressure at a discrete number of acoustic field points. Optimization of the sensor design parameters continues until the objective function in the nonlinear optimization is reduced to the same level as that obtained in path 1. At this point, the maximum control authority of the actuator(s) over the acoustic field has been obtained. Further optimization of the PVDF sensor design parameters cannot increase the level of acoustic control achieved with the given optimally located control actuator(s).

\section{B. Nonlinear optimal control algorithm}

Whether the piezoelectric actuator location(s) is being optimized or the PVDF error sensor size(s) and shape(s) are being optimized the format of the design variables is identical. As a result of this observation, the initial formulation of the nonlinear optimization algorithm will be presented in generic form for optimization of a rectangular element. This element can be the actuator or the error sensor depending on whether path 1 or path 2 of Fig. 1 is chosen. A schematic of the simply supported plate configured with a rectangular patch whose location and size are to be optimized is presented in Fig. 3. The dimensions of the rectangular element in the $x$ and $y$ direction of the coordinate system will be denoted $w_{x_{i}}$ and $w_{y_{i}}$, respectively. The spatial coordinates of the center of the rectangular element will be denoted $\bar{x}_{i}$ and $\bar{y}_{i}$ with respect to the $x-y$ axis. These variable parameters are independent of whether the rectangular element is termed an actuator or a sensor.

If path 1 of the algorithm is chosen, the total radiated sound pressure can be written as a function of the coordinates of the control actuator(s) as well as the control voltage(s) as follows:

$$
p_{t}=p_{t}\left(\bar{x}_{a_{i}}, \bar{y}_{a_{i}}, w_{x_{a_{i}}}, w_{y_{a_{i}}}, V_{a_{i}}\right),
$$

where the subscript $a$ indicates coordinates of an actuator, and the subscript $i$ refers to the $i$ th actuator. In this section of the algorithm, the actuator dimension was fixed, and only the location(s) of the actuator(s) was optimized. In addition, one should recognize that the optimal control voltage, $V_{a}$ is a function of the coordinates and dimensions of the actuator as well. Rewriting Eq. (21) as a function of the variables included in the optimization, we obtain

$$
p_{t}=p_{t}\left(\bar{x}_{a_{i}}, \bar{y}_{a_{i}}, V_{a_{i}}\left(\bar{x}_{a_{i}}, \bar{y}_{a_{i}}\right)\right) \text {. }
$$

Now, if path 2 of the optimization algorithm is chosen, and hence the PVDF error sensor size(s) and location(s) are optimized, the total pressure can be expressed as follows: where the subscript $s$ indicates coordinates of the PVDF error sensor, and the subscript $i$ refers to the $i$ th sensor. At this point in the design approach, the optimal actuator location(s) has been computed. However, at each step in the algorithm the optimal control voltage(s) to the actuator(s)

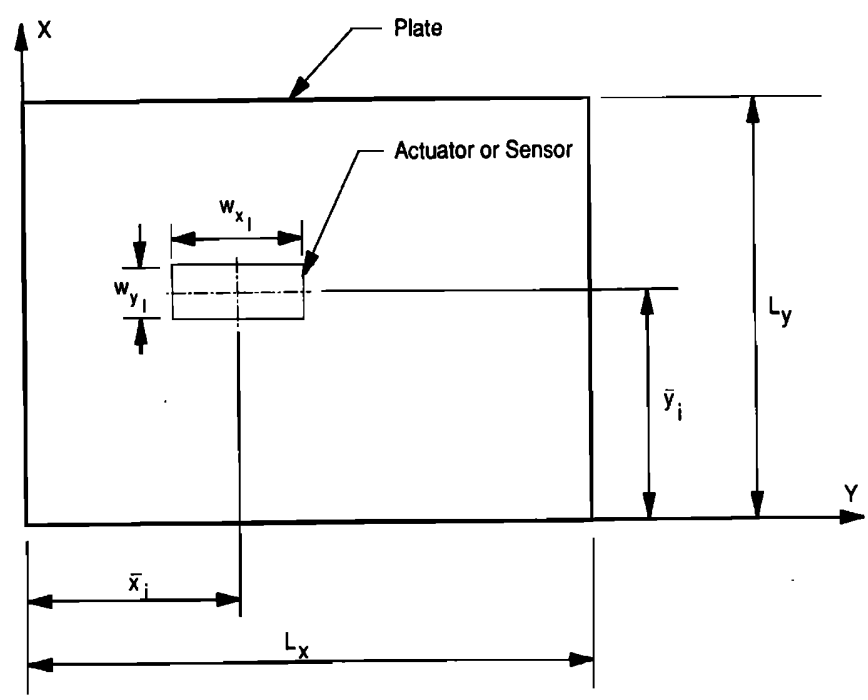

FIG. 3. Schematic of optimization variables for rectangular sensor or actuator. 
is updated, resulting in the minimization of the error sensor response for the current sensor design parameters. As a result, the control voltage( $s$ ) to the actuator(s) is now a function of the sensor design parameters:

$$
p_{t}=p_{t}\left(\bar{x}_{s_{i}}, \bar{y}_{s_{t}}, w_{x_{s_{i}}}, w_{y_{s_{i}}}, V_{a}\left(\bar{x}_{s_{i}}, \bar{y}_{s_{i}}, w_{x_{s_{i}}}, w_{y_{s_{t}}}\right)\right) \text {. }
$$

All design variables can be constrained to meet physical limitations such as actuator size(s), actuator voltage(s), and sensor size(s).

\section{Objective function}

The desired goal is to locate the actuator(s) and sensor(s) in such a way that the total far-field sound radiation from the structure is minimal. Based on this goal, the logical choice for an objective function is one based on the radiated far-field sound power or a reasonable estimate of this quantity. Wang et al. chose the integral of the square of radiated sound pressure over a hemisphere of the structure as the objective function. ${ }^{10}$ This objective function can be formulated as follows:

$$
\Phi_{p}=\int_{0}^{2 \pi} \int_{0}^{\pi / 2}\left|p_{t}\right|^{2} \sin (\theta) d \theta d \phi .
$$

In view of practical implementation, Wang and Fuller proposed a finite sum of the mean-square radiated sound pressures measured by a limited number of microphones as an approximation to the above objective function. ${ }^{18}$ This objective function can be represented by the following summation:

$$
\Psi_{p}=\sum_{i=1}^{N_{\text {mic }}}\left|p_{t_{i}}\left(R_{i}, \theta_{i}, \phi_{i}\right)\right|^{2} .
$$

In implementing Eq. (25), a reasonable number of pressure sensors should be chosen such that the global estimate of the objective function is not lost; however, the number of pressure sensors should not be so great as to significantly increase the computational time of the program. More work is needed to overcome this limitation.

\section{Physical constraints}

The optimal design parameters for both the actuator(s) and sensor(s) were constrained to meet physical limitations such as plate boundaries as well as limitations on control voltage(s) to the actuator(s). If path 1 of the algorithm was chosen, the piezoelectric actuator(s) must be constrained to the boundaries of the plate. For the case of multiple actuators, the design parameters were further constrained to prevent overlap between actuators. Finally, the allowed control voltage was constrained to $400 \mathrm{~V}$ peak to peak in accordance with previous tests conducted demonstrating the point of failure on identical actuators. ${ }^{15}$ Constraints are listed below. ies:

(1) Constrain piezoelectric actuator to plate boundar-

$$
\begin{aligned}
& \bar{x}_{a_{i}}-w_{x_{a_{i}}} / 2 \geqslant 0, \\
& \bar{x}_{a_{i}}+w_{x_{a_{i}}} / 2 \leqslant L_{x}, \\
& \bar{y}_{a_{i}}-w_{y_{a_{i}}} / 2 \geqslant 0, \\
& \bar{y}_{a_{i}}+w_{y_{a_{i}}} / 2 \leqslant L_{y} .
\end{aligned}
$$

(2) Constrain overlap between control actuators: ${ }^{17}$

$$
\begin{aligned}
& {\left[\left(\bar{x}_{a_{(i+1)}}-\bar{x}_{a_{i}}\right)^{2}+\left(\bar{y}_{a_{(i+1)}}-\bar{y}_{a_{i}}\right)^{2}\right]^{1 / 2}} \\
& \quad-\frac{1}{2}\left[\left(w_{x_{a_{i}}}^{2}+w_{y_{a_{i}}}^{2}\right)^{1 / 2}-\left(w_{x_{a_{(i+1)}}^{2}}^{2}+w_{y_{a_{(i+1)}}}^{2}\right)^{1 / 2}\right]>0 .
\end{aligned}
$$

(3) Constrain voltage to piezoelectric actuator:

$$
\left|V_{a_{i}}\right| \leqslant 200 \quad \text { (volt p-p). }
$$

In path 2 of the algorithm, the sensor size and location must be contrained to that of the plate boundaries. In addition, for the case of multiple sensors, the solution was constrained to prevent overlap between sensors. All sensor constraints are listed below.

(1) Constrain PVDF error sensor dimensions to that of the plate:

$$
\begin{aligned}
& 0 \leqslant w_{x_{s_{i}}} \leqslant L_{x} / 2, \\
& 0 \leqslant w_{y_{s_{t}}} \leqslant L_{y} / 2 .
\end{aligned}
$$

(2) Constrain PVDF error sensor to plate boundaries:

$$
\begin{aligned}
& \bar{x}_{s_{i}}-\omega_{x_{s_{i}}} / 2 \geqslant 0, \\
& \bar{x}_{s_{i}}+w_{x_{s_{i}}} / 2 \leqslant L_{x}, \\
& \bar{y}_{s_{i}}-w_{y_{s_{i}}} / 2 \geqslant 0, \\
& \bar{y}_{s_{i}}+w_{y_{s_{i}}} / 2 \leqslant L_{y} .
\end{aligned}
$$

(3) Constrain overlap between PVDF error sensors: ${ }^{17}$

$$
\begin{aligned}
& {\left[\left(\bar{x}_{s_{(i+1)}}-\bar{x}_{s_{i}}\right)^{2}+\left(\bar{y}_{s_{(i+1)}}-\bar{y}_{s_{i}}\right)^{2}\right]^{1 / 2}} \\
& \quad-\frac{1}{2}\left[\left(w_{x_{s_{i}}}^{2}+w_{y_{s_{i}}}^{2}\right)^{1 / 2}-\left(w_{x_{s_{(i+1)}}}^{2}+w_{y_{s_{(i+1)}}^{2}}^{2}\right)^{1 / 2}\right]>0 .
\end{aligned}
$$

\section{Review of optimization}

Upon determining the constraints on the design parameters, the optimization routine is implemented. An IMSL subroutine named NOONF, which solves a general nonlinear programming problem, was implemented to compute the optimal solution for both path 1 and path 2 of Fig. 1. The gradient of the design parameters with respect to the objective function was estimated within the successive quadratic programming algorithm by means of an IMSL subroutine called CDGRD, which applies the central finite difference method to approximate the gradient. The general constrained optimization problem can be stated mathematically with the objective function: ${ }^{21}$

$$
f(\mathbf{x})=f\left(x_{1}, x_{2}, \ldots, x_{n}\right),
$$

subject to the equality constraints

$$
h_{j}(\mathbf{x})=0, \quad \text { for } j=1, m_{e},
$$

and the equality constraints

$$
g_{j}(\mathbf{x})>0, \quad \text { for } j=1, m_{(e+1)}, m
$$

where $m_{e}$ is the number of equality constraints and $m$ is the total number of constraints. Both the objective function $f$ and the constraint functions $h_{j}$ and $g_{j}$ are assumed to be continuously differentiable. The solution technique is based on the iterative formulation and solution of quadratic programming subproblems. The subproblems are obtained by 
using a quadratic approximation of the Lagrangian (which is a function formed in terms of the objective function and the constraint functions) and linearizing the constraints as follows:

$$
\min _{d \in \mathscr{R}^{n}} \frac{1}{2} \mathrm{~d}^{T}\left[H_{k}\right] \mathrm{d}+\nabla f\left(\mathbf{x}_{k}\right)^{T} \mathrm{~d}
$$

subject to

$$
\nabla h_{j}\left(\mathbf{x}_{k}\right)^{T} \mathrm{~d}+h_{j}\left(\mathbf{x}_{k}\right)=0, \quad \text { for } j=1, m_{e}
$$

and

$$
\nabla g_{j}\left(\mathbf{x}_{k}\right)^{T} \mathbf{d}+g_{j}\left(\mathbf{x}_{k}\right)=0, \quad \text { for } j=1, m_{(e+1)}, m,
$$

where $\left[H_{k}\right]$ is a positive definite approximation of the Hessian matrix (which is a matrix composed of the second partial derivatives of the objective function with respect to each of the design variables) and $\mathbf{x}_{k}$ is the current iterate. If $\mathbf{d}_{k}$ is chosen as the solution of the subproblem, then a line search can be used to find the new point $x_{k+1} \cdot{ }^{22}$

$$
\mathbf{x}_{k+1}=\mathbf{x}_{k}+\lambda \mathbf{d}_{k}, \quad \lambda \in(0,1],
$$

such that a "merit function" will have a lower function at the new point. The augmented Lagrange function is used as the merit function for this problem. ${ }^{23}$ This iteration process is continued until the accuracy test is passed, otherwise the design parameters are updated and the algorithm is executed for another consecutive iteration.

\section{Summary of procedure}

The previous sections outlining the linear quadratic optimal control approach as well as the nonlinear optimization algorithm were written with optimization of multiple actuator/sensor configurations in mind. For the purpose of this study, only one actuator and sensor location were considered since this was sufficient for demonstrating the advantages afforded with optimization. The optimization approach implemented in path 1 and path 2 of the algorithm are identical with the exception that the actuator size(s) is not optimized in path 1 . This choice was made due to dimensions of available actuator material as well as maximum voltage limitations imposed as actuator size decreases.

\section{DISCUSSION OF TEST CASE}

To demonstrate the advantages afforded with optimization of actuator and sensor locations, a test case with a disturbance at an excitation frequency of $550 \mathrm{~Hz}$ was chosen. This excitation frequency corresponds to off-resonance excitation as can be ascertained from the tabulated resonant frequencies for the simply supported plate. Both analytical and experimental values of the resonant frequencies for the first nine modes of the plate are listed in Table I. The plate chosen for this study was constructed from steel and measured $380 \times 300 \times 1.96 \mathrm{~mm}$. The boundary conditions of this plate have been established in prior studies to be consistent with the simply supported condition. ${ }^{11-13,16} \mathrm{~A}$ picture of the test plate is presented in Fig. 4. As illustrated, four actuators and three PVDF sensors are configured on the plate surface. The first three actuators and the first two PVDF error sensors have been used in previous studies and were left on the plate as a basis for comparison. The fourth actuator and the third
TABLE I. Theoretical and measured plate resonant frequencies $f_{m n}$.

\begin{tabular}{ccc}
\hline \hline $\begin{array}{c}\text { Mode } \\
(m, n)\end{array}$ & $\begin{array}{c}\text { Theoretical } \\
\text { frequency } \\
(\mathbf{H z})\end{array}$ & $\begin{array}{c}\text { Measured } \\
\text { frequency } \\
(\mathbf{H z})\end{array}$ \\
\hline$(1,1)$ & 87.6 & 87 \\
$(2,1)$ & 188.5 & 187 \\
$(1,2)$ & 249.7 & 247 \\
$(2,2)$ & 350.6 & 347 \\
$(3,1)$ & 356.5 & 352 \\
$(3,2)$ & 518.6 & 517 \\
$(1,3)$ & 519.9 & 520 \\
$(4,1)$ & 591.7 & 590 \\
$(2,3)$ & 620.7 & 615 \\
$(4,2)$ & 753.8 & 750 \\
$(3,3)$ & 788.7 & 779 \\
\hline \hline
\end{tabular}

PVDF sensor, labeled OPT-PZT and OPT-PVDF, were located in the optimal configuration based upon the analytical results for an excitation frequency of $550 \mathrm{~Hz}$ for the above system.

The coordinates of the lower left corner and the upper right corner of the optimally located actuator are $(311,232)$ $\mathrm{mm}$ and $(349,263) \mathrm{mm}$, respectively. The remaining actuators pictured were located at antinodes of the $(3,3)$ mode of the plate to increase control authority when implementing multi-channel control. Coordinates of the lower left corner and upper right corner of the optimally sized and located error sensor are $(13,0) \mathrm{mm}$ and $(265,22) \mathrm{mm}$, respectively. The disturbance was generated with a shaker located on the back of the plate (not pictured) at spatial coordinates of $(240,130) \mathrm{mm}$. The input force was measured with a Kistler force transducer which was attached between the stinger of the shaker and the plate.

Experiments were performed in the anechoic chamber at Virginia Polytechnic Institute and State University that has dimensions $4.2 \mathrm{~m} \times 2.2 \mathrm{~m} \times 2.5 \mathrm{~m}$ and a cutoff frequency of $250 \mathrm{~Hz}$. The simply supported plate was centered in a baffle made of particle board extending across the widest dimension of the chamber to replicate the appropriate acoustic boundary conditions. The baffled simply supported plate

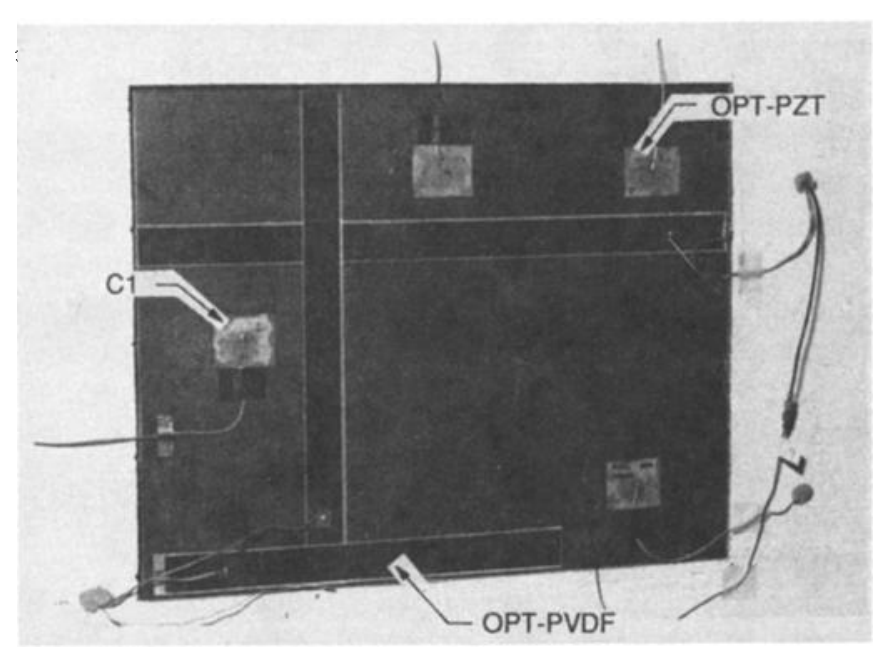

FIG. 4. Piecture of test plate with actuators and sensors. 


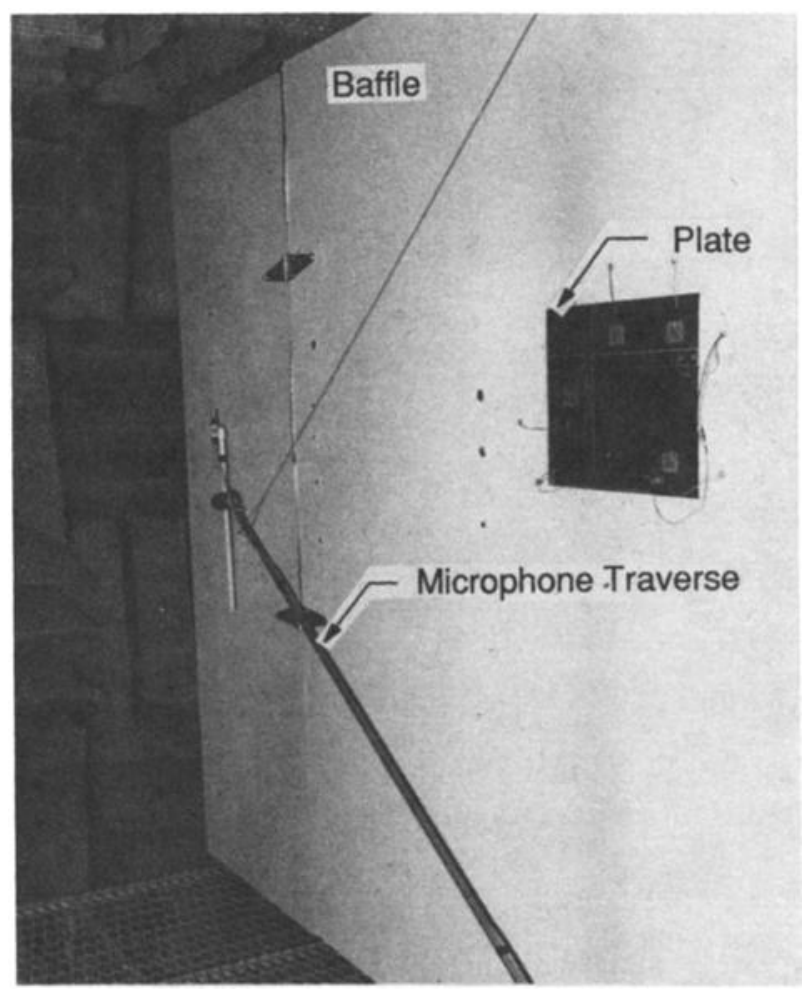

FIG. 5. Picture of baffled simply supported plate.

is pictured in Fig. 5 with the traversing microphone used to map the directivity pattern of the acoustic response about the horizontal center line of the plate. For all tests conducted, the acoustic response was measured in $9^{\circ}$ increments about the baffle for a total of 21 measurement coordinates. This test rig is used for a variety of studies at VPI \& SU to evaluate different control actuators and error sensors for active structural acoustic control (ASAC) and has been outlined in previous work by Clark and Fuller. ${ }^{12}$ For all experiments conducted, control was achieved with a narrow band, multi-channel version of the filtered- $x$ adaptive LMS algorithm which was implemented on a TMS320C25 digital signal processing board resident in an AT computer. The algorithm was written such that the user could select the number of inputs and outputs, each ranging from one to three for - control implementation. This control algorithm has been outlined previously by Clark and Fuller, ${ }^{12,13}$ and a rigorous discussion of the algorithm can be found in previous work by Elliot et al. ${ }^{24}$

Several test cases were conducted to demonstrate the performance and efficiency advantages resulting from optimization, and for each experiment conducted, the analytical results were computed for comparison. Results comparing the controlled acoustic response achieved with the three nonoptimal actuators are compared to those implementing control with the optimally located actuator. Test cases utilizing the optimal control actuator and the three nonoptimal control actuators were conducted implementing three acoustic error sensors (microphones) at a radius of $1.6 \mathrm{~m}$ and angles for $\theta$ and $\phi$ of $\left(45^{\circ}, 0^{\circ}\right),\left(45^{\circ}, 180^{\circ}\right)$, and $\left(0^{\circ}, 0^{\circ}\right)$ as referenced in Fig. 2. Upon completing these test cases, the piezoelectric actuator configured in the optimal location was used in conjunction with the PVDF error sensor optimally sized and located. Results from this single channel control case were compared to those from the previously performed multi-channel control cases. Optimization was restricted to a single channel in this study since no significant advantage was gained by optimizing the location of multiple control actuators for the particular test case chosen.

\section{RESULTS}

\section{A. Evaluation of optimal actuator location}

The results presented in Fig. 6(a) and (b) were used to demonstrate the level of acoustic control that can be achieved with a single optimally located piezoelectric actuator. Error sensors used in this test case were three microphones oriented at a radius of $1.6 \mathrm{~m}$ and angles of $\left(45^{\circ}, 0^{\circ}\right)$, $\left(45^{\circ}, 180^{\circ}\right)$, and $\left(0^{\circ}, 0^{\circ}\right)$, which will be designated $45^{\circ},-45^{\circ}$, and $0^{\circ}$ in the figures displaying the acoustic directivity pattern for convenience. Three tests were conducted to demonstrate the advantages afforded with the optimally located actuator. In the first case, a single, nonoptimally located actuator (denoted C1) was used to achieve control. In the sec-

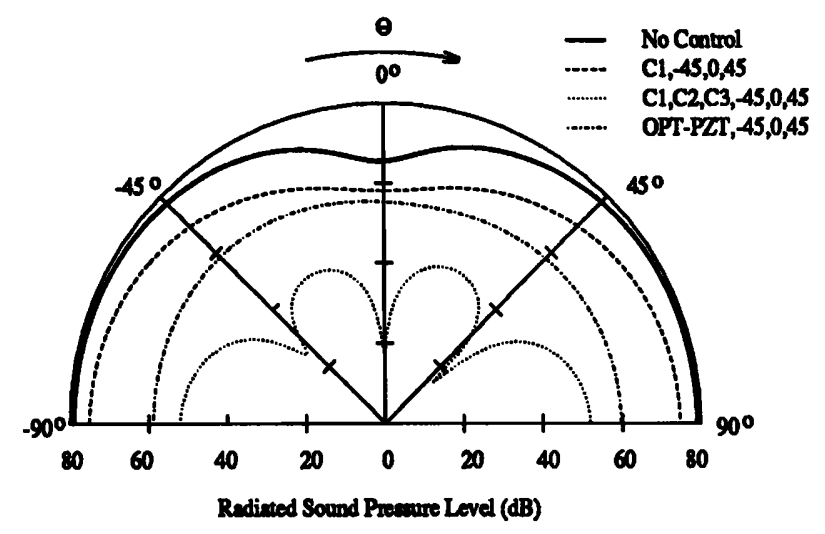

(a) Predicted acoustic directivity pattern

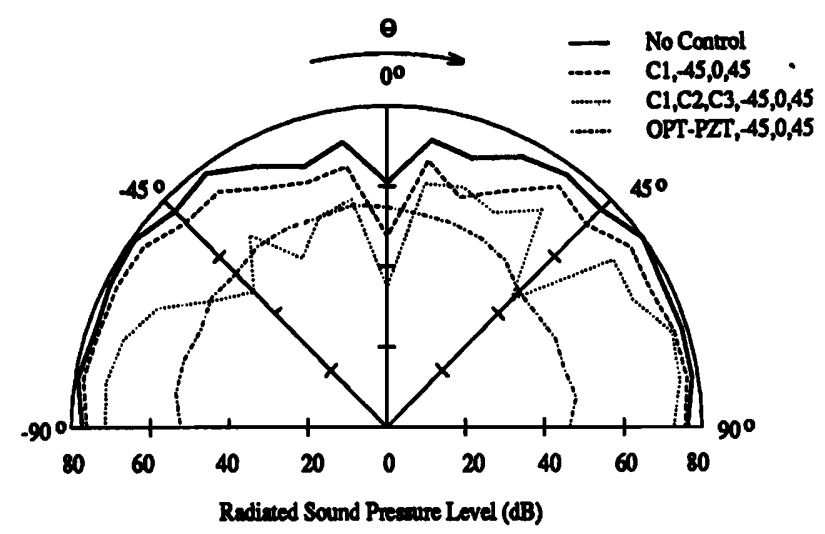

(b) Measured acoustic directivity pattern

FIG. 6. Comparison of theory and experiment for optimization of actuator location. 
ond case, all three nonoptimally located actuators (denoted $\mathrm{C} 1, \mathrm{C} 2, \mathrm{C} 3$ ) were utilized to achieve control, and finally, the optimally located actuator (denoted OPT-PZT) was used to achieve acoustic control. Both analytical and experimental results are compared in Fig. 6(a) and (b), respectively. Analytical results predict an increase in acoustic attenuation on the order of $10 \mathrm{~dB}$ when implementing the optimally located actuator as compared to the nonoptimally located control actuator as observed in Fig. 6(a). Experimental results of Fig. 6(b) confirm this predicted result with even more significant attenuation observed along the axis near the baffle. Microphones place out of the plane of the traversing microphone confirmed the global level of acoustic attenuation. Discrepancies noted between the measured and predicted acoustic directivity pattern are likely due to the fact that the baffle implemented was of finite dimensions as compared to the infinite baffle assumed in computing the predicted response of the simply supported plate.

The final test case implementing microphones as error sensors was conducted with all three nonoptimally located control actuators. As indicated in Fig. 6(a), significant attenuation is predicted at the error sensors, and approximately 20-30 dB of acoustic attenuation is predicted in the residual field (i.e., locations other than that of error microphones). Comparing the experimental results of Fig. 6(b) to the predicted results, the "notches" at the error microphones were measured; however, the residual response is much poorer than predicted. In general, the level of acoustic attenuation near the baffle is significantly less than that predicted. This phenomenon could be due to dipole effects resulting from the finite dimension of the baffle. In addition, the error between the predicted and measured structural response of the plate has been shown to increase with increasing number of actuators used to drive the structure for the approximate dynamic model of the piezoelectric actuator implemented in this study. ${ }^{16}$ Thus errors in computing the acoustic response of the structure based upon the approximate dynamic model of the piezoelectric actuator contribute to the discrepancies observed in the multi-actuator control case. The exact cause of this deviation between theory and experiment for the acoustic response of the plate is however unknown. Based on experimental results, levels of acoustic attenuation achieved with the optimally located control actuator rival those achieved with three nonoptimal control actuators and certainly surpasses that of a single non-optimally located control actuator.

\section{B. Evaulation of optimal actuator/sensor configuration}

The final set of tests were chosen to evaluate the levels of acoustic attenuation achieved when the PVDF error sensor is used as the cost function as opposed to the three microphones previously discussed. In the first test case, a nonoptimally located control actuator (denoted C1) is used in conjunction with the optimally oriented PVDF error sensor (denoted OPT-PVDF) to compare results with the optimal actuator/sensor configuration. The predicted level of attenuation for the nonoptimal actuator is approximately $5 \mathrm{~dB}$ as illustrated in Fig. 7(a), which is comparable with that predicted when using microphone error sensors as illustrat-

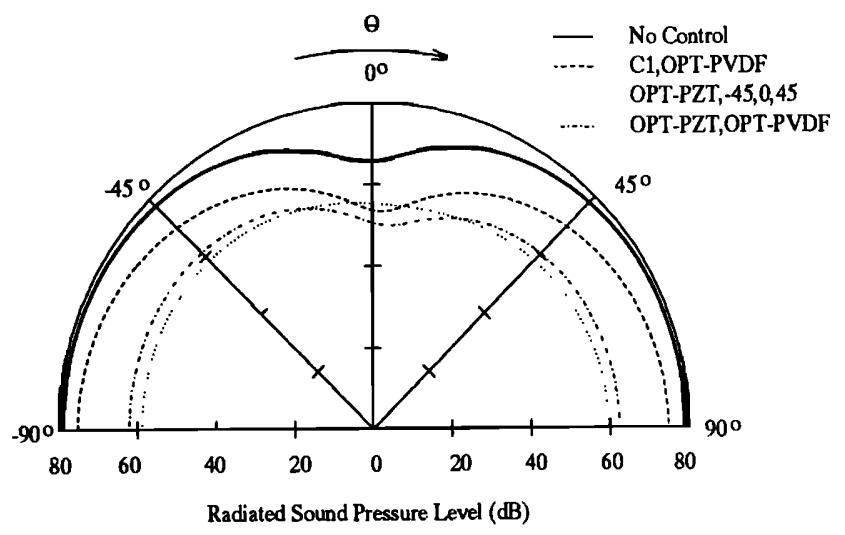

(a) Predicted acoustic directivity pattern

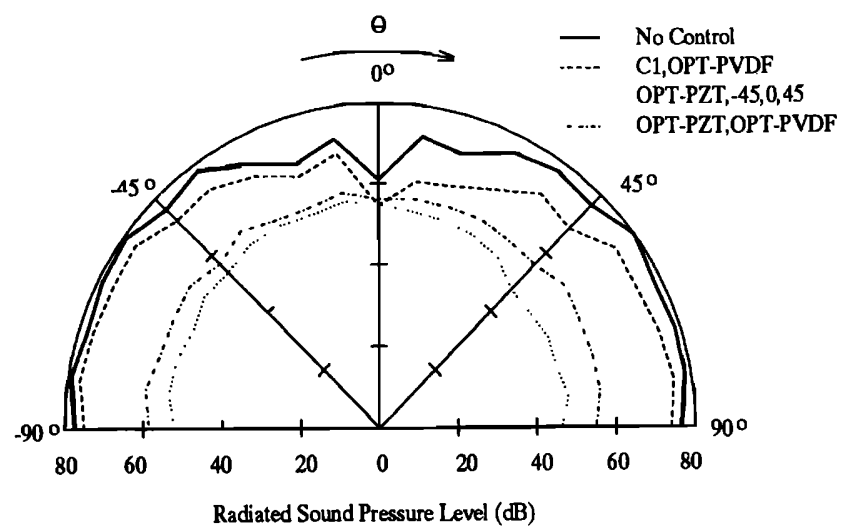

(b) Measured acoustic directivity pattern

FIG. 7. Comparison of theory and experiment for optimization of actuator and sensor location.

ed in Fig. 6(a). When the optimal actuator/sensor configuration (denoted OPT-PZT,OPT-PVDF) is implemented, the predicted level of acoustic attenuation increases by approximately $10 \mathrm{~dB}$ as illustrated in Fig. 7(a). Experimental results, presented in Fig. 7(b), for this test case yield similar trends with even greater levels of attenuation, approximately $20 \mathrm{~dB}$, observed near the baffle. Microphones placed out of the plane of the traversing microphone confirmed that the level of sound pressure attenuation was global for the cases presented. Levels of acoustic attenuation increase significantly when using the optimally configured actuator/sensor pair. This is due to the increase in control authority over the acoustic response of the plate gained through optimization of the actuator location.

In the final test case, the acoustic response when implementing microphones with the optimally located actuator (denoted OPT-PZT,45,0, - 45) is compared to the acoustic response resulting from control with the optimal actuator/ sensor pair. Both the predicted and measured acoustic re- 
sponse of Fig. 7(a) and (b) yield similar trends. In general, the level of acoustic attenuation for the optimal actuator/ sensor configuration is the same as that measured and predicted when the optimal actuator was used in conjunction with the three microphone error sensors. In addition, the total power radiated from the structure upon achieving control was predicted from the analytical results with each error sensor configuration. Control implementation with the optimal actuator/sensor configuration resulted in attenuation of acoustic power within $0.1 \mathrm{~dB}$ of that predicted when the optimally located actuator was used in conjunction with microphone error sensors.

\section{Physical interpretation of optimal actuator/sensor configuration}

As indicated from the analytical and experimental results, a significant level of acoustic attenuation was achieved with a single optimally located piezoelectric control actuator and a single optimally sized and positioned PVDF error sensor. Before proceeding, with the conclusions, an interpretation of the optimal actuator/sensor configuration is in order.

Referring to the picture of the test plate in Fig. 4, one observes that the optimally located control actuator is in the upper right-hand corner of the plate. Since the nature of the disturbance (point force excitation) is different from that of the piezoelectric actuator (line moments induced at the boundaries of the actuator), co-location is not a problem. Considering the modal response of the plate, the optimal actuator location appears logical. For off-resonance re- sponse, as the modal density of the plate increases (i.e., number of modes contributing significantly to the structural response) the control actuator must be able to efficiently couple into the structural modes which radiate sound. As the actuator draws closer to the boundaries of the plate, the number of modes that can be excited increases. For example, an actuator capable of coupling into all structural modes of the plate would be very small and located in the corner of the plate. However, this would require an extremely large control voltage to elicit response due to the high structural input impedance at this location, and is therefore not practical. In addition, for structural-acoustic control the objective is not necessarily to control all modes on the plate, rather to control those modes contributing to the far-field sound radiation. Excitation of modes with poor radiation efficiency can be conveniently reduced by positioning the actuator over or near the nodal line of the given mode. In other words, the position and dimension of the control actuator creates a spatial window on the surface of the structure which can be used to "filter" the structural modes excited by the actuator in both relative phase and magnitude, thereby creating a response which most efficiently couples into the modes radiating sound.

A similar interpretation can be used upon viewing the optimally located and sized PVDF error sensor depicted in Fig. 4. The sensor creates a spatial window defined by its dimension and position on the surface of the plate. Since the goal is to eliminate the radiating acoustic modes, sensor design parameters are optimal when the "filter" created by the spatial window of the sensor observes only those modes con-

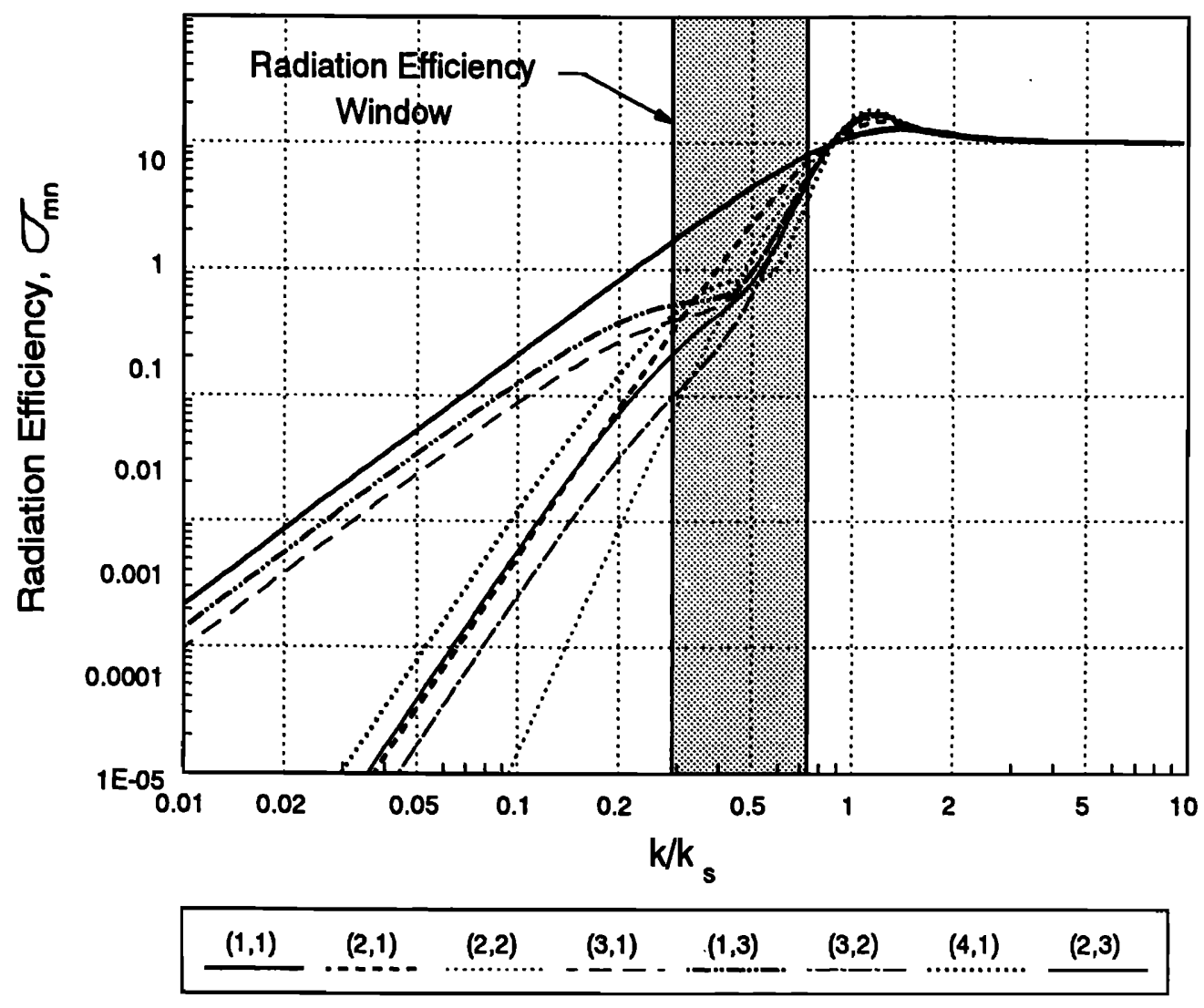

FIG. 8. Radiation efficiency of selected structural modes. 
tributing to the far-field sound radiated. In other words, the optimal sensor configuration for the given test case can be viewed as a superconsic wave-number filter, in which the subsonic components are not observed.

Due to the modal density of the off-resonance response of the plate at the chosen excitation frequency, an analysis of the model response of the structure simply complicates the discussion. Each structural mode has a radiation efficiency which is computed for the driving frequency of $550 \mathrm{~Hz}$. A plot of the radiation efficiency for each structural mode of the simply supported plate used in this study is presented in Fig. 8 as a function of the ratio of the acoustic wave number $k$ to the structural wave number $k_{s}$ of the chosen mode. The "radiation efficiency window" depicted in Fig. 8 illustrates the range of the radiation efficiencies for each structural mode contributing to the response of the plate for an excitation frequency of $550 \mathrm{~Hz}$ which corresponds to an acoustic wave number of $10.2(1 / \mathrm{m})$ in air. The modes with higher modal indices such as the $(2,3)$ mode have larger structural wave numbers $\left(k_{s}\right)$ and thus smaller values of $k / k_{s}$ while the modes with lower modal indices such as the $(1,1)$ mode have smaller structural wavenumbers and hence larger values of $k / k_{s}$. As observed in Fig. 8, the radiation efficiency of each structural mode depends upon the value of $k / k_{s}$ for the chosen mode; however, less separation between the radiation efficiency of modes is observed in this operating region as compared to the relative radiation efficiency of the same modes for $k / k_{s}<0.2$

In addition to the observation that the radiation efficiencies of the modes contributing to the acoustic response are of similar orders of magnitude, modal coupling between the more efficient modes contributes significantly to the overall sound radiation in this region of operation. Thus simply looking at the individual radiation efficiencies of each structural mode does not clearly define the overall sound radiation characteristics of the structure. To better illustrate the structural-acoustic response of the plate, a plot of the theoretical time-averaged acoustic intensity before and after control is presented in Fig. 9(a) and (b), respectively. (The time-averaged acoustic intensity was computed as outlined by Petterson ${ }^{25}$ and is reviewed in the Appendix.) As illustrated, a significant reduction in the overall sound power (approximately $15 \mathrm{~dB}$ ) is observed in Fig. 9(b) after applying control with the optimally configured PZT actuator and implementing the optimally designed PVDF error sensor. Comparing the acoustic intensity before and after control, one observes that the majority of the sound radiation occurs at the boundaries of the plate. Thus the position of the optimal PVDF error sensor appears logical. The rectangular sensor is located along the edge of the plate where significant levels of sound radiation occurs.

The uncontrolled plate can thus be viewed as an edge radiator since the majority of the sound power flows from the edges of the plate as discussed in previous work by Maidanik. ${ }^{26}$ The optimal location for the PVDF strain sensor is on the lower boundary of the plate, corresponding to the location of the edge radiation. Had the sensor extended the full length of the plate in the $x$ direction, the $(4,1)$ mode would not have been observed due to the symmetry of the

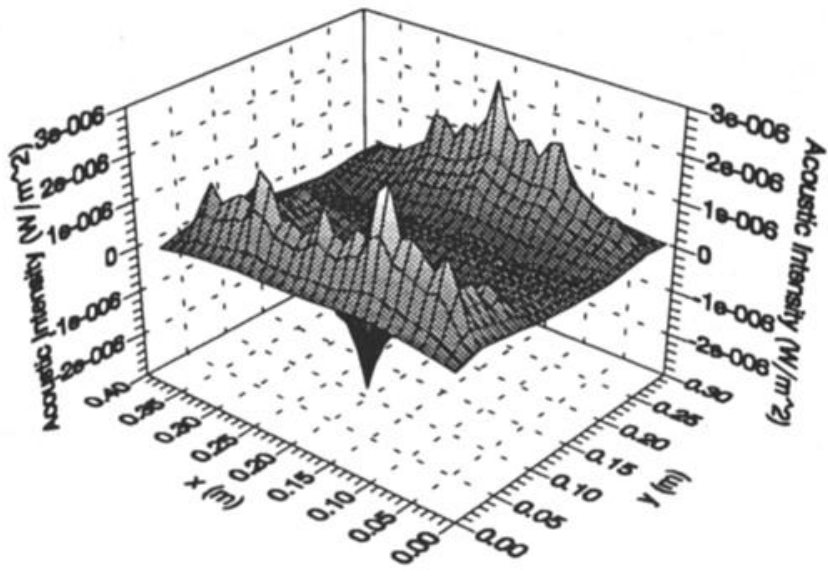

(a) Before control

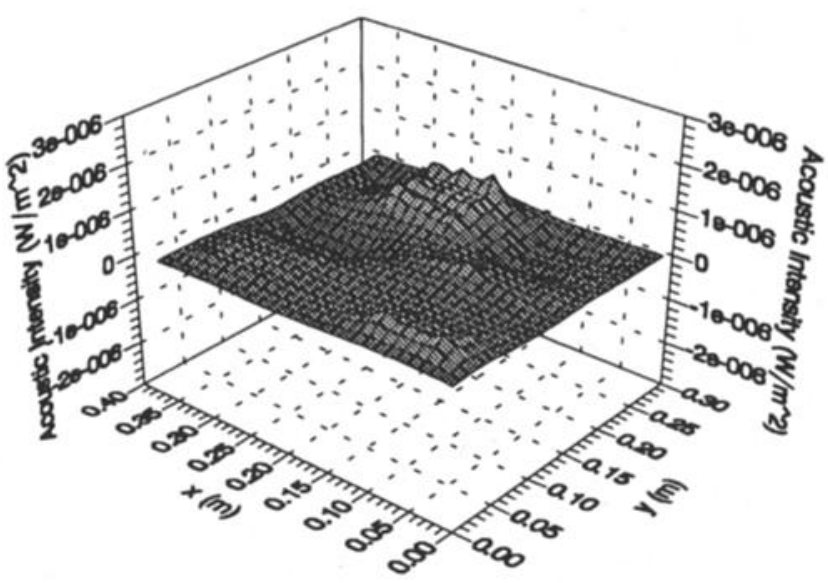

(b) After control

FIG. 9. Time-averaged acoustic intensity before and after control.

modal response in the $x$ direction. The length of the sensor in this dimension dictates the weighting of the $(4,1)$ mode, while the location of the sensor relates to the edge radiating $(1,3)$ mode. This physical interpretation emphasizes the importance of optimizing both the shape and location of the PVDF error sensor for structural acoustic control.

\section{CONCLUSIONS}

The purpose of this work was to experimentally confirm results from previous analytical studies by Wang and Fuller ${ }^{17}$ suggesting that a single optimally located piezoelectric actuator can be used to obtain significant levels of acoustic attenuation from a baffled simply supported plate excited by a harmonic disturbance. In addition, a method for optimizing the size and location of a rectangular strip of polyvinylidene fluoride (PVDF) is outlined for replacing microphone error sensors in the control approach. For both cases, an off-resonance test .case was chosen since the structural and acoustic response is dependent upon the complex interaction of multiple modes.

The optimal design of the PZT control actuator location alone resulted in significant levels of global sound power reduction, rivaling that of the nonoptimal multi-actuator control applications on the plate studied. In addition, the 
optimal design of a structural PVDF error sensor for structural-acoustic control resulted in a sensor whose dimensions and position were such that the spatial window created by the sensor resulted in a control function proportional to the sound radiation from the structure (i.e., a supersonic wavenumber filter). For the preliminary design case presented, a single optimally configured PZT actuator and PVDF error sensor were implemented to demonstrate global sound power reduction rivaling that achieved with multiple control actuators and multiple microphone error sensors placed in the acoustic field.

While the results presented were for the optimal design of a single control actuator and a single structural error sensor, the multiple-input/multiple-output design optimization procedure was outlined. As with all optimal design techniques, a model of the objective function (the structural acoustic response in this case) is required for the design. For this preliminary study, the design optimization was based upon an analytical model; however, the model can be obtained from finite element techniques or experimental methods for more complex structures. Based on the results of this study, a technique for choosing appropriate structural error sensors and control actuators for minimizing far-field sound radiation is outlined for a simple test structure. The ingredients for implementation of an adaptive structure for active structural acoustic control appear to be available considering control authority achieved with piezoelectric actuators and sensing possibilities afforded with distributed polyvinylidene fluoride (PVDF).

\section{ACKNOWLEDGMENTS}

The authors gratefully acknowledge the support of this work by DARPA and the Office of Naval Research under grant ONR-N00014-88-K-0721.

\section{APPENDIX: COMPUTATION OF TIME-AVERAGED ACOUSTIC INTENSITY}

The time-averaged acoustic intensity is computed based upon a finite difference approximation of the gradient of the pressure computed at two acoustic field points in the direction chosen for obtaining the power flow. ${ }^{26}$ Due to Euler's inviscid equation of motion in a fluid, the velocity of the fluid can be obtained from the density of the medium and the gradient of the pressure for harmonic motion as follows:

$$
j \omega \rho_{0} \mathbf{v}=-\nabla p,
$$

where $\rho_{0}=$ density, $v=$ velocity of fluid, $p=$ acoustic pressure. Hence the pressure is averaged to determine the approximate pressure between the two acoustic field points as follows.

$$
p_{12} \simeq\left(p_{1}+p_{2}\right) / 2
$$

and velocity is estimated between the two field points by the finite difference technique as follows:

$$
v_{12} \simeq-\frac{p_{2}-p_{1}}{\rho_{0} \Delta_{12}},
$$

where $\Delta_{12}=$ distance between acoustic field points, $p_{12}=$ pressure between acoustic field points, $v_{12}=$ velocity between acoustic field points. Both $p_{1}$ and $p_{2}$ are in general complex quantities. Based upon these approximations of velocity and pressure, the time-averaged acoustic intensity can be obtained as follows:

$$
\bar{I}_{12} \approx \frac{\left|p_{1}\right|\left|p_{2}\right|}{2 \rho_{0} \omega \Delta_{12}} \sin \left(\phi_{1}-\phi_{2}\right),
$$

where $\bar{I}_{12}$ is the time-averaged acoustic intensity between the two field points and $\phi_{1}$ and $\phi_{2}$ are the phase angles associated with the respective pressures $p_{1}$ and $p_{2}$.

${ }^{\prime}$ E. F. Crawley and J. de Luis, "Use of piezoelectric actuators as elements of intelligent structures," AIAA J. 25 (10), 1373-1385 (1989).

${ }^{2} J$. L. Fanson and J. C. Chen, "Structural control by the use of piezoelectric active members," in Proceedings of NASA / DOD Control-Structures Interaction Conference, NASA CP-2447, Part II (1986).

${ }^{3}$ T. Bailey and J. E. Hubbard, "Distributed piezolectric-polymer active vibration control of a cantilevered beam," AIAA J. Guidance Control 6 (5), 605-611 (1986).

${ }^{4}$ S. E. Burke and J. Hubbard, "Active vibration control of a simply supported beam using a spatially distributed actuator," IEEE Control. Syst. Mag., 25-30 (1987).

${ }^{5}$ J. M. Plump, J. E. Hubbard, and T. Bailey, "Nonlinear control of a distributed system: Simulation and experimental results," J. Dynan. System Meas. and Control. 109, 133-139 (1987).

${ }^{6} \mathrm{C}$. K. Lee, W. W. Chiang, and T. C. O'Sullivan, "Piezoelectric modal sensors and actuators achieving critical damping on a cantilever plate," AIAA Paper, No. 89-1390 (1989).

${ }^{7}$ D. W. Miller, S. A. Collins, S. P. Peltzman, "Development of spatially convolving sensors for structural control applications," AIAA Paper, 901127-CP, 2283-2297 (1990).

${ }^{8}$ C. K. Lee and F. C. Moon, "Modal sensors/actuators," ASME JAM 57, 434-441 (1990).

${ }^{9}$ C. R. Fuller, C. H. Hansen, and S. D. Snyder, "Active control of structurally radiated noise using piezoceramic actuators," Proceedings of InterNoise 89, 509-511 (1989).

${ }^{10} \mathrm{~B}$. T. Wang, E. K. Dimitriadis, and C. R. Fuller, "Active control of structurally radiated noise using multiple piezoelectric actuators," Proceedings of AIAA SDM Conference, AIAA Paper 90-1172-CP, Long Beach, California (1990).

"'R. L. Clark and C. R. Fuller, "Experiments on active control of structurally radiated sound using multiple piezoceramic actuators," J. Acoust. Soc. Am. 91(5) 3313-3320 (1992).

${ }^{12}$ R. L. Clark and C. R. Fuller, "Active structural acoustic control with adaptive structures including wavenumber considerations," J. Intell. Mater. Systems Structures 2(3), 431-452 (1991).

${ }^{13}$ R. L. Clark and C. R. Fuller, "Modal sensing of efficient acoustic radiators with PVDF distributed sensors in active structural acoustic approaches," J. Acoust. Soc. Am. 91, 3321-3329 (1992).

${ }^{14}$ E. K. Dimitriadis, C. R. Fuller, and C. A. Rogers, "Piezolectric actuators for distributed vibration excitation of thin plates," J. Vib. Acoust., 100107 (1991).

${ }^{15}$ R. L. Clark, C. R. Fuller, A. L. Wicks, "Characterization of multiple piezolectric actuators for structural excitation," J. Acoust. Soc. Am. 90, 346-357 (1991).

${ }^{16}$ R. L. Clark, M. R. Fleming, and C. R. Fuller, "Piezoelectric actuators for distributed vibration excitation of thin plates: A comparison between theory and experiment," accepted for publication in J. Vib. Acoust. (1992).

${ }^{17}$ B. T. Wang, R. A. Burdisso, and C. R. Fuller, "Optimal placement of piezoelectric actuators for active control of sound radiation from elastic plates," Proceedings of Noise-Con 91, 267-275 (1991).

${ }^{18} \mathbf{B}$. T. Wang and C. R. Fuller, "Near-field pressure, intensity and wavenumber distributions for active structural control of plate radiation: Theoretical analysis," accepted for publication in J. Acoust. Soc. Am. (1992).

${ }^{19} \mathrm{~L}$. Meirovitch Analytical Methods in Vibrations (Macmillian, New York, 1967), pp. 179-185.

${ }^{20} \mathrm{~F}$. Fahy, Sound and Structural Vibration (Academic, Orlando, FL, 1987), pp. 61-63.

${ }^{21}$ J. Arora, Introduction to Optimum Design (McGraw-Hill, New York, 1989), p. 111.

${ }^{22}$ User's Manual: IMSL Math/Library, Version 1.1, 897-902 (1989).

${ }^{23} \mathrm{~K}$. Schittkowski, "NLPQL: A FORTRAN subroutine solving con- 
strained nonlinear programming problems," edited by Clyde L. Monma, Ann. Operations Res. 5, 485-500 (1982).

${ }^{24}$ S. J. Elliott, I. M. Stothers, and P. A. Nelson, "A multiple error LMS algorithm and its application to the active control of sound and vibration," IEEE Trans. Acoustic Speech Signal Process. ASSP-35, 1423-
1434 (1987).

${ }^{25} \mathrm{O}$. K. Petterson, "A procedure for determining the sound intensity distribution close to a vibration surface," J. Sound Vib. 66(4), 626-629 (1979).

${ }^{26} \mathrm{G}$. Maidanik, "Response to ribbed panels to reverberant acoustic fields," J. Acoust. Soc. Am. 34, 809 (1962). 